\title{
Implementação CUDA dos Kernels NPB
}

\author{
Gabriell Alves de Araujo ${ }^{1}$, Dalvan Griebler ${ }^{1,2}$, Luiz G. Fernandes ${ }^{1}$ \\ ${ }^{1}$ Escola Politécnica, Grupo de Modelagem de Aplicações Paralelas (GMAP), \\ Pontifícia Universidade Católica do Rio Grande do Sul (PUCRS), Porto Alegre, Brasil. \\ ${ }^{2}$ Laboratório de Pesquisas Avançadas para Computação em Nuvem (LARCC), \\ Faculdade Três de Maio (SETREM), Três de Maio - RS - Brasil \\ \{gabriell.araujo, dalvan.griebler\}@acad.pucrs.br, luiz.fernandes@pucrs.br
}

\begin{abstract}
Resumo. NAS Parallel Benchmarks (NPB) é um conjunto de benchmarks utilizado para avaliar hardware e software, que ao longo dos anos foi portado para diferentes frameworks. Concernente a GPUs, atualmente existem apenas versões OpenCL e OpenACC. Este trabalho contribui com a literatura provendo a primeira implementação CUDA completa dos kernels do NPB, realizando experimentos com carga de trabalho inédita e revelando novos fatos sobre o NPB.
\end{abstract}

\section{Introdução}

Unidades de Processamento Gráfico (GPUs) têm sido utilizadas como alternativa para computação de alto desempenho, pois oferecem capacidade massiva de paralelismo a baixo custo. No entanto, o paralelismo das GPUs é um desafio para os desenvolvedores [Xu et al. 2015]. Benchmarks têm assumido papel relevante no desenvolvimento, contribuindo para a avaliação de arquiteturas e técnicas de programação [Seo et al. 2011]. NAS Parallel Benchmarks (NPB) [Bailey et al. 1994] é um conjunto de benchmarks baseado em dinâmica de fluidos que possui pertinência científica e têm se destacado em pesquisas de GPUs nos últimos anos.

No entanto, ao observar a literatura, é possível perceber algumas lacunas em aberto. Os principais trabalhos são relativamente antigos, não foram realizados experimentos com cargas de trabalho grandes, baixa expectativa de aceleração foi relatada, e também não existe disponível uma versão CUDA dos kernels do NPB. O que é relatado pela literatura como impedimento para o avanço de pesquisas [Tian et al. 2016].

As contribuições deste trabalho visam preencher estas lacunas identificadas. Assim, o trabalho apresenta a primeira versão CUDA completa dos kernels do NPB. Realiza experimentos com a carga de trabalho Classe $\mathrm{C}$, não explorada nos trabalhos anteriores por limitações das abordagens e arquiteturas. Compara a versão CUDA deste trabalho com as versões OpenCL [Seo et al. 2011] e OpenACC [Xu et al. 2015] do estado-da-arte. Avalia desempenho, consumo de memória e quantidade de linhas. Novos fatos são revelados sobre o NPB, alcançando melhores resultados que os registros da literatura, atualizando as expectativas de aceleração através de nova implementação e experimentos.

\section{Implementação}

A implementação paralela foi realizada a partir da versão C++ do NPB [Griebler et al. 2018]. Foram adotados princípios de desenvolvimento tais como, implementar os kernels de GPU da forma mais simples possível, evitando fluxos complexos e divergências de branches, coalescer acessos na memória, utilizar a hierarquia de memória evitando acessos na memória global e implementar paralelismo de GPU apenas em trechos que oferecem boa performance na GPU, observando overheads e custos de transferência de memória. 
CG realiza aproximações de valores e foi desenvolvido com o objetivo de avaliar computações irregulares [Bailey et al. 1994]. A computação mais intensiva de CG consiste na multiplicação de submatrizes não estruturadas, uma lista de tarefas onde cada tarefa acessa diferentes locais da memória e realiza quantidades diferentes de computações. A maneira mais adequada de abordar este problema é isolar as computações irregulares. Para isto, cria-se um grupo de threads para cada uma das tarefas. As computações de cada tarefa são dividas entre as threads do grupo, e a execução é então realizada de forma regular em cada grupo de threads.

EP visa estimar a capacidade de ponto-flutuante da arquitetura alvo e por isso possui poucas dependências [Bailey et al. 1994]. EP possui um laço global onde cada iteração gera um conjunto de números aleatórios e após calcula desvios Gaussianos. Para aplicar o paralelismo, cada iteração é atribuída a um grupo de threads e cada thread do grupo calcula um subconjunto dos números e desvios. As threads reutilizam a memória até calcularem todos os resultados. Isto é necessário pelo motivo de que os subconjuntos consomem muita memória da GPU.

FT resolve equações diferenciais de números complexos [Bailey et al. 1994]. As computações mais intensivas são as funções que computam Transformação Rápida de Fourier [Bailey et al. 1994] nas dimensões $x, y$ e $z$ dos vetores. Estas funções possuem muitos laços aninhados, dependências de dados, divergências de branches e baixo grau de paralelismo. Para implementar o benchmark de forma a extrair bom desempenho na GPU, dividi-se cada uma destas funções em três estágios, eliminando dependências de dados, divergências de branches e aumentando o grau de paralelismo. O primeiro estágio realiza cópia de dados, o segundo estágio realiza o processamento dos dados e o terceiro estágio escreve os dados na saída. Para o cálculo da função no eixo x, adicionalmente é necessário transformar o padrão de acesso para coalescer a memória.

IS estima a velocidade e comunicação de números inteiros através de computações de ordenação [Bailey et al. 1994]. Diferente dos outros benchmarks, IS é constituído por sequências simples de laços, e o mapeamento para as threads de GPU se resume em criar uma thread para cada iteração. No entanto, as computações são pouco intensivas, o grau de paralelismo é baixo, e são necessárias sincronizações entre as sequências de laços, características que impactam de forma negativa o desempenho das GPUs.

MG é uma implementação multi-grid simplificada [Bailey et al. 1994]. MG possui quatro funções de computação intensiva, interp, rprj3, psinv e resid. As funções interp e rprj3, possuem laços aninhados, onde a computação interna destes laços trata-se de uma sequência de laços. Para aplicar o paralelismo na GPU, os laços externos são combinados em único laço, onde cada iteração é associada a um grupo de threads. Junta-se a sequência interna de laços em um único laço, e as iterações deste são divididas entre as threads do grupo. As funções psinv e resid possuem organização similar a interp e $r p r j 3$, por esse motivo são paralelizados da mesma forma, porém, os laços internos não podem ser combinados em um único laço. Portanto, os grupos de threads precisam executar sequências de laços em vez de um único laço.

\section{Experimentos}

Os experimentos foram realizados em uma máquina equipada com um processador Intel Xeon E5-2620 2.0 GHz, 16 Gigabytes de RAM, e uma GPU NVIDIA Titan X Pascal com 3584 CUDA Cores e 12 Gigabytes de memória dedicada. O sistema operacional utilizado foi o Ubuntu 14.04 LTS. Os softwares utilizados foram CUDA 10, GCC-9, 
OpenCL 1.1, e OpenACC 2.5. Utilizou-se a flag de compilação -O3 em todos os benchmarks. Cada experimento foi repetido 10 vezes para a computação das métricas. Como carga de trabalho foi utilizada a Classe C do NPB. Os tempos de execução obtiveram desvio padrão irrelevante e são apresentados na Figura 1(a) O consumo de memória da GPU é apresentado na Figura 1(b), A quantidade de linhas de código necessária em cada implementação é apresentada na Figura 2 e na Tabela 1. A implementação CUDA deste trabalho é apresentada como NPB-CUDA, os trabalhos do estado-arte com OpenCL e OpenACC são apresentados respectivamente como NPB-OpenCL [Seo et al. 2011] e NPB-OpenACC [Xu et al. 2015]. NPB-OpenCL e NPB-OpenACC precisaram da flag de compilação -mcmodel=large e do comando ulimit -s unlimited para permitir mais memória na stack, pois implementam consumo de memória de forma ineficiente, alocando grande vetores multi-dimensionais nesta região de memória.

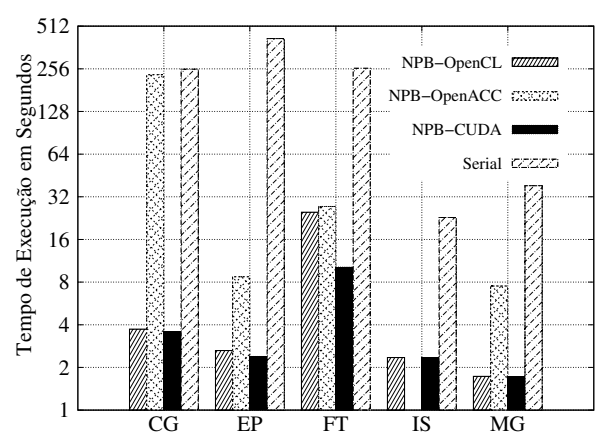

(a) Tempo de Execução.

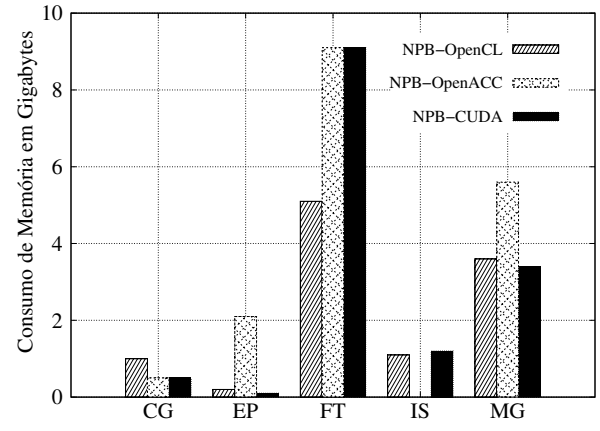

(b) Consumo de Memória da GPU.

Figura 1. Resultados dos Experimentos com a Classe C.

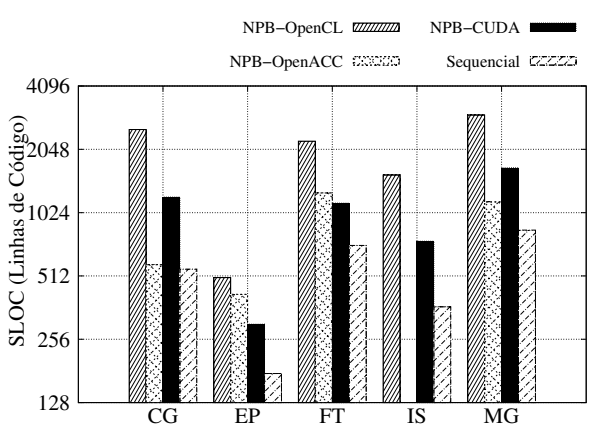
Tabela 1. Aumento de linhas de código em relação ao código sequencial.

\begin{tabular}{|c|c|c|c|}
\hline Kernel & NPB-OpenCL & NPB-OpenACC & NPB-CUDA \\
\hline CG & $360,87 \%$ & $4,89 \%$ & $119,93 \%$ \\
\hline EP & $185,80 \%$ & $138,07 \%$ & $71,59 \%$ \\
\hline FT & $213,15 \%$ & $77,62 \%$ & $58,88 \%$ \\
\hline IS & $323,56 \%$ & - & $105,21 \%$ \\
\hline MG & $252,13 \%$ & $36,05 \%$ & $97,75 \%$ \\
\hline
\end{tabular}

Figura 2. Total em linhas de código.

Em CG, NPB-CUDA obteve 71.5 de speedup, desempenho obtido através do isolamento das computações irregulares. O desempenho foi $4.48 \%$ melhor que NPB-OpenCL por explorar maior paralelismo através de kernels de GPU concorrentes. NPB-OpenACC apresentou baixo desempenho por não isolar as computações irregulares. Em EP, NPB-CUDA alcançou 171.5 de speedup, por meio do paralelismo de grão fino implementado. O resultado foi $9.98 \%$ melhor que NPB-OpenCL. NPB-OpenCL possui mais conflitos de memória e misses, recebendo impacto na aceleração. NPB-OpenACC teve menor desempenho por utilizar estratégia de grão grosso. Em FT, NPB-CUDA atingiu 25.3 de speedup, $143.26 \%$ melhor que NPB-OpenCL. Os trabalhos NPB-OpenCL e NPB-OpenACC não eliminaram dependências de dados e divergências de branches, e mantiveram baixo grau de paralelismo. Por isso, o desempenho foi significativamente inferior. Em IS, não foi 
possível realizar experimentos com NPB-OpenACC, pois os autores não disponibilizaram a implementação. O speedup de NPB-CUDA foi 9.8, muito próximo a NPB-OpenCL. Em MG, NPB-CUDA também apresentou desempenho próximo a NPB-OpenCL, decorrência da similaridade entre as abordagens. NPB-OpenACC atingiu menor desempenho pelo motivo de que a implementação possui overhead de sincronizações entre GPU e CPU.

O desempenho dos benchmarks varia significativamente. EP roda exclusivamente na GPU. Os demais benchmarks precisam de sincronizações entre CPU e GPU. FT e MG possuem divergências de caminhos que não podem ser removidas e possuem diferentes padrões de acessos. IS possui baixo grau de paralelismo. O consumo de memória das implementações NPB-CUDA foi próximo aos melhores resultados entre NPB-OpenCL e NPB-OpenACC, com exceção de FT, onde o trabalho NPB-OpenCL implementa reutilização de memória. Quanto a quantidade necessária de linhas de código para aplicar o paralelismo, NPB-OpenCL apresenta número superior a NPB-CUDA. NPB-OpenACC possui mais linhas de código que NPB-CUDA apenas em EP e FT.

\section{Conclusão}

Este trabalho apresentou a primeira versão CUDA completa dos kernels do NPB e foi comparado aos trabalhos OpenCL e OpenACC do estado-da-arte. Os resultados demonstraram aceleração considerável nos benchmarks. Adicionalmente, a implementação deste trabalho obteve melhor desempenho em três aplicações. As investigações deste trabalho contribuem para o domínio de dinâmica de fluidos, avaliando o comportamento em GPUs. Como trabalhos futuros, pretende-se implementar versão multi-GPU dos kernels do NPB, paralelizar as pseudo-aplicações e também utilizar outros frameworks de GPUs.

\section{Referências}

Bailey, D. H., Barszcz, E., Barton, J. T., Browning, D. S., Carter, R. L., Fatoohi, R. A., Frederickson, P. O., Lasinski, T. A., Simon, H. D., Venkatakrishnan, V., and Weeratunga, S. K. (1994). The NAS Parallel Benchmarks RNR-94-007. Technical report, NASA Advanced Supercomputing Division.

Griebler, D., Loff, J., Mencagli, G., Danelutto, M., and Fernandes, L. G. (2018). Efficient NAS Benchmark Kernels with C++ Parallel Programming. In 26th Euromicro International Conference on Parallel, Distributed and Network-Based Processing (PDP), PDP'18, pages 733-740, Cambridge, UK. IEEE.

Seo, S., Jo, G., and Lee, J. (2011). Performance Characterization of the NAS Parallel Benchmarks in OpenCL. In 2011 IEEE International Symposium on Workload Characterization (IISWC), pages 137-148.

Tian, X., Xu, R., Yan, Y., Chandrasekaran, S., Eachempati, D., and Chapman, B. (2016). Compiler Transformation of Nested Loops for General Purpose GPUs. Concurrency and Computation: Practice and Experience, 28(2):537-556.

Xu, R., Tian, X., Chandrasekaran, S., Yan, Y., and Chapman, B. (2015). NAS Parallel Benchmarks for GPGPUs Using a Directive-Based Programming Model. In Brodman, J. and Tu, P., editors, Languages and Compilers for Parallel Computing, pages 67-81, Cham. Springer International Publishing. 